\title{
Discovery of a highly potent and selective degrader targeting hematopoietic prostaglandin D synthase via in silico design
}

Hidetomo Yokoo ${ }^{1}$, Norihito Shibata ${ }^{2 *}$, Akinori Endo ${ }^{3}$, Takahito Ito ${ }^{1}$, Yuta Yanase ${ }^{1,4}$, Yuki Murakami ${ }^{1,4}$, Kiyonaga Fujii ${ }^{5}$, Yasushi Saeki ${ }^{3}$, Mikihiko Naito ${ }^{6 *}$, Kosuke Aritake $^{7 *}$, and Yosuke Demizu $^{1,4 *}$

${ }^{1}$ Division of Organic Chemistry, National Institute of Health Sciences, 3-25-26 Tonomachi, Kawasaki-ku, Kawasaki-shi, Kanagawa 210-9501, Japan

2Division of Biochemistry, National Institute of Health Sciences, 3-25-26 Tonomachi, Kawasaki-ku, Kawasaki-shi, Kanagawa 210-9501, Japan

${ }^{3}$ Protein Metabolism Project, Tokyo Metropolitan Institute of Medical Science, 2-1-6 Kamikitazawa, Setagaya-ku, Tokyo 156-8506, Japan

${ }^{4}$ Graduate School of Medical Life Science, Yokohama City University, 1-7-29 Suehirocho, Tsurumi-ku, Yokohama-shi, Kanagawa 230-0045, Japan

${ }^{5}$ Laboratory of Analytical Chemistry, Daiichi University of Pharmacy, 22-1 Tamagawa-machi, Minami-ku, Fukuoka-shi, Fukuoka 815-8511, Japan

${ }^{6}$ Laboratory of Targeted Protein Degradation, Graduate School of Pharmaceutical Sciences The University of Tokyo, 7-3-1 Hongo, Bunkyo-ku, Tokyo 113-0033, Japan

${ }^{7}$ Laboratory of Chemical Pharmacology, Daiichi University of Pharmacy, 22-1 Tamagawamachi, Minami-ku, Fukuoka-shi, Fukuoka 815-8511, Japan

* : corresponding author

E-mail: demizu@nihs.go.jp

E-mail: n-shibata@nihs.go.jp

E-mail: miki-naito@g.ecc.u-tokyo.ac.jp 
E-mail: k-aritake@daiichi-cps.ac.jp 


\begin{abstract}
Hematopoietic prostaglandin D synthase (H-PGDS) is an attractive target for the development of therapeutic agents for Duchenne muscular dystrophy (DMD) and other $\mathrm{H}-$ PGDS-related diseases. We have recently developed the H-PGDS degrader PROTAC(HPGDS)-1, which is a chimeric molecule in which TFC-007 (that binds to H-PGDS) and pomalidomide (that binds to cereblon [CRBN]) were conjugated to the PEG5 linker. Herein, using a docking simulation of the ternary complex of the H-PGDS degrader, H-PGDS, and CRBN, we have succeeded in developing PROTAC(H-PGDS)-7, a new H-PGDS degrader that does not contain a linker. PROTAC(H-PGDS)-7 showed potent and selective degradation activity $\left(\mathrm{DC}_{50}=17.3 \mathrm{pM}\right)$, and potent suppression of prostaglandin $\mathrm{D}_{2}\left(\mathrm{PGD}_{2}\right)$ production in KU812 cells. Additionally, in a DMD model using $m d x$ mice with cardiac hypertrophy, PROTAC(H-PGDS)-7 showed better inhibition of inflammatory cytokines than TFC-007. PROTAC(H-PGDS)-7 is expected to be a promising candidate for the treatment of DMD and other H-PGDS-related diseases.
\end{abstract}




\section{Introduction}

Targeted protein degradation by proteolysis targeting chimeras (PROTACs) is one of the most exciting and emerging modalities for drug discovery and biological discovery. PROTACs are bifunctional molecular glues that function to link individual ligands for E3 ligase with the target protein, eliciting ectopic ubiquitination, and resulting in the degradation of the target protein via the ubiquitin-proteasome system (UPS) $)^{1,2}$. Advances in targeted protein degradation strategies have accelerated over the past decade, and many PROTACs have been developed for the treatment of cancer and neurodegenerative diseases. Notably, ARV110, which is a PROTAC-based degrader for the androgen receptor, is currently in a Phase 2 clinical study for metastatic castration-resistant prostate cancer ${ }^{2}$.

The main strategy for the development of PROTAC compounds is to investigate the appropriate combination of ligands for E3 ligase and the target protein, and modify the linking moiety, which can vary widely ${ }^{3}$. Additionally, structural elucidation of the PROTAC with the E3 ligase and target protein is important for rational design and optimization of the PROTAC ${ }^{4-}$ ${ }^{6}$. However, it is generally difficult to obtain a crystal structure of the ternary complex. In recent years, there have been a few reports of in silico PROTAC design ${ }^{7-10}$, but in many cases, it is necessary to synthesize a multitude of PROTACs with different types and lengths of linkers by trial and error to identify potent PROTACs.

Hematopoietic prostaglandin D synthase (H-PGDS) catalyzes the synthesis of prostaglandin $\mathrm{D}_{2}\left(\mathrm{PGD}_{2}\right)$. The overproduction of $P G D_{2}$ is involved in a variety of diseases, including allergic diseases ${ }^{11,12}$, physiological sleep $^{13}$, and Duchenne muscular dystrophy (DMD) $)^{14,15}$, and thus makes H-PGDS a promising therapeutic target. We have very recently developed the chimeric molecule PROTAC(H-PGDS)-1, which is linked between TFC-007 (that binds to H-PGDS) and pomalidomide (that binds to cereblon [CRBN]) via the PEG5 linker (Fig. 1a) ${ }^{16}$. PROTAC(H-PGDS)-1 potently degraded the H-PGDS protein via the UPS and effectively suppressed PGD2 production. 
In this study, to optimize the degradation activity of PROTACs targeting H-PGDS, a docking simulation of the ternary complex of PROTAC(H-PGDS), H-PGDS, and CRBN was performed (Fig.s 1b and 1c). As a result, it was found that PROTAC(H-PGDS)-7 without any PEG linker was able to form the ternary complex, which showed highly potent, selective, and effective H-PGDS degradation activity. Furthermore, in an in vivo study, PROTAC(H-PGDS)7 showed better activity than the H-PGDS inhibitor TFC-007. The results from this study suggested that a docking simulation would be useful for the design and optimization of PROTACs if a crystal structure of the target protein and E3 ligase with their respective ligands is available. 


\section{Results}

Docking simulation of ternary complex and design the linker.

The ternary complex of PROTAC(H-PGDS)-1 with the PEG5 linker, H-PGDS, and CRBN was initially calculated using the docking software suite MOE PROTAC-Modeling Tools in which the calculation method was implemented ${ }^{7,8}$. Molecular dynamics (MD) simulations were subsequently performed using the stable structure obtained from the above calculation to clarify the behavior of the ternary complex in solution. A stable conformation showed that the PEG5 linker in PROTAC(H-PGDS)-1 was bent in an S-shape structure (Fig. 1b). To analyze the modest length of linker, we performed a docking study of each ternary complex with designated PROTAC(H-PGDS)-3-6 with shorter lengths of the PEG linker than PEG5, and PROTAC(H-PGDS)-7 linking pomalidomide directly to TFC-007. Reasonable ternary complexes in which each PROTAC(H-PGDS) designed with different length of the PEG linker fills the cavity between H-PGDS and CRBN were obtained (Extended data Fig.1), notably, even in the case of PROTAC(H-PGDS)-7 (Fig. 1c). In each calculated structure, the hydrogen bond between an oxygen atom of the imide moiety of pomalidomide and the $\mathrm{H} 380$ of CRBN was retained after MD simulation. The $\pi-\pi$ interaction between the pyrimidine moiety of TFC-007 and the W104 of H-PGDS was retained after MD simulation of PROTAC(H-PGDS)-4, 6 and 7 (Extended data Fig.2). These results suggest that PROTAC(H-PGDS) with a shorter linker tends to form a more stable ternary complex. Additionally, the difference of the linker length, i.e., PROTAC(H-PGDS)-1 versus 7, changed the relationship of the orientation between H-PGDS and CRBN in the stable ternary complex (Extended data Fig.3a, S3b), which possibly affects the approach of the entire E3 ligase complex to H-PGDS.

\section{Reduction activity toward H-PGDS protein of PROTAC(H-PGDS)-1, 3-10.}

To examine the effect of the linker length of the PROTACs against H-PGDS on the reduction activity toward H-PGDS protein, PROTAC(H-PGDS)-1, 3-7 were synthesized (see 
Supporting Information). The effect of the synthesized PROTAC(H-PGDS) on the H-PGDS protein was evaluated using KU812 cells and a 6-h incubation (Fig. 2a). Effective reduction of H-PGDS protein by the PROTAC(H-PGDS)s was observed at concentrations $\geq 10 \mathrm{nM}$, and as the linker was shortened, the activity was increased. Among the compounds, PROTAC(H-PGDS)-7, which is directly linked to pomalidomide and TFC-007, showed the strongest activity for reduction of H-PGDS protein with half-maximal degradation concentration ( $\mathrm{DC}_{50}$ ) values of $26.3 \mathrm{pM}$ for $6 \mathrm{~h}$ and $17.3 \mathrm{pM}$ for $24 \mathrm{~h}$, respectively (Fig. $2 \mathrm{~b}, 2 \mathrm{c}$ ). Similar protein reduction activity for PROTAC(H-PGDS)-7 was also observed in MEG-01s cells expressing H-PGDS protein (Extended data Fig.4). The reduction activity was not observed for PROTAC(H-PGDS)-VH (Extended data Fig.5), in which the TFC-007 derivative is linked to the von Hippel-Lindau (VHL) ligand $\mathrm{VH} 032$ to recruit $\mathrm{VHL}$ as an E3 ligase via an ethylenediamine linker whose length between TFC-007 and the E3 ligase ligand mimics that of the directly linked PROTAC(H-PGDS)-7.

TFC-007 and another H-PGDS inhibitor F092 contain the same $N$-phenyl-5pyrimidinecarboxamide moiety. According to the co-crystal structure of the H-PGDS protein with the ligand F092, which is the TFC-007 mimetic (PDB: 5YWX), the moiety faces to the inside of the H-PGDS protein (Extended data Fig.6). Therefore, the $\mathrm{N}$-carbonyl piperazino piperidine moiety of PROTAC(H-PGDS)-7 would not be required for binding to the H-PGDS protein and might function as a linker. On the basis of the above structural information, to investigate the structure-activity relationship of PROTAC(H-PGDS)-7, especially the binding site between TFC-007 and the pomalidomide, PROTAC(H-PGDS)-9 was designed by elimination of the piperazino- $N$-carbonyl moiety of PROTAC(H-PGDS)-7. PROTAC(HPGDS)-10 was also designed by changing the piperazine moiety of PROTAC(H-PGDS)-7 to an ethylenediamine to investigate the need for rigidity of the binding site. To confirm that those modification on TFC-007 moiety in PROTAC(H-PGDS)-7 maintain the binding affinities to H-PGDS, the binding affinities to H-PGDS of PROTAC(HPGDS)-9 and PROTAC(HPGDS)-10 was measured by using a competitive binding assay with a fluorescence probe 
and those compounds showed comparable activity as TFC-007 (Extended data Fig.7). However, PROTAC(H-PGDS)-9 did not induce a reduction of the H-PGDS protein (Extended data Fig.8). From a docking simulation of PROTAC(H-PGDS)-9, the distance between the pyrimidine moiety of TFC-007 and W104 of H-PGDS was not stable during 100ps and that between the imide moiety of pomalidomide and H380 of CRBN was stable as longer than that of PROTAC(H-PGDS)-7 during MD simulation (Extended data Fig.3c, 3d), suggesting that the ternary complex of PROTAC(H-PGDS)-9, H-PGDS, and CRBN may not be stable. While, PROTAC(H-PGDS)-10 showed lower activity for reduction of the H-PGDS protein. Those results suggested that the piperazino- $N$-carbonyl moiety of PROTAC(H-PGDS)-7 would function as a linker and its rigidity increased the reduction activity.

\section{Selective degradation of H-PGDS via ubiquitin-proteasome system.}

To investigate whether PROTAC(H-PGDS)-7 is also a degrader for the H-PGDS protein via the UPS similar to PROTAC(H-PGDS)-1 ${ }^{16}$, we examined the effect of PROTAC(H-PGDS)-7 on turnover of the H-PGDS protein. Treatment with the protein synthesis inhibitor cycloheximide in KU812 cells dramatically decreased the levels of H-PGDS protein in the PROTAC(H-PGDS)-7-treated cells, compared with its retention in the control cells (Extended data Fig.10a). Furthermore, the levels of H-PGDS mRNA in the KU812 cells were not affected by PROTAC(H-PGDS)-7 (Extended data Fig.10b). These results indicated PROTAC(H-PGDS)-7 induces the degradation of H-PGDS protein.

To explore the mechanism of PROTAC(H-PGDS)-7-induced degradation of the H-PGDS protein, we first confirmed the combination of TFC-007 and pomalidomide (100 pM each) did not effectively decrease the amount of H-PGDS protein (Fig. 3a). A competition assay using an excess amount of pomalidomide to inhibit the chimera PROTAC(H-PGDS)-7 diminished the protein degradation activity of PROTAC(H-PGDS)-7 (Fig. 3b). We also developed PROTAC(H-PGDS)-8 by conjugating TFC-007 with an N-methylated pomalidomide, which shows reduced binding affinity to CRBN. While TFC-007, PROTAC(HPGDS)-7, and 
PROTAC(H-PGDS)-8 showed similar affinity toward H-PGDS with $\mathrm{IC}_{50}$ values of $0.17,0.14$, and $0.17 \mu \mathrm{M}$, respectively (Fig. 3c), PROTAC(H-PGDS)-8 did not show degradation activity of the H-PGDS protein (Fig. 3d). These data indicate that CRBN binding was required for the H-PGDS protein degradation by PROTAC(H-PGDS)-7. To investigate the involvement of the UPS in the degradation of H-PGDS protein by PROTAC(H-PGDS)-7, we used the proteasome inhibitor MG132 and the ubiquitin-activating enzyme inhibitor MLN7243. The PROTAC(H-PGDS)-7-induced degradation of H-PGDS protein was suppressed by both inhibitors (Fig. 3e). Those results suggested that the degradation of H-PGDS protein requires the UPS.

To investigate the specificity of PROTAC(H-PGDS)-7 for H-PGDS degradation, we performed proteome profiling of KU812 cells that were treated for $6 \mathrm{~h}$ with $100 \mathrm{pM}$ or $10 \mathrm{nM}$ PROTAC(H-PGDS)-7 and compared them to DMSO-treated cells. Protein levels were quantified using tandem mass tag (TMT) labeling and mass spectrometry (MS) (Fig. 4a). HPGDS levels were reduced to $30.8 \%\left(P=3.31 \times 10^{-5}\right)$ at 100 pM PROTAC(H-PGDS)-7 and to $12.9 \%\left(P=1.23 \times 10^{-7}\right)$ at $10 \mathrm{nM}$ PROTAC(H-PGDS)-7 compared with DMSO-treated cells (Fig. 4b and Supplementary Dataset). The reduction of H-PGDS by 100 pM PROTAC(H-PGDS)-7 was restored by MG132 (Fig. 4b). Strikingly, of the 8,184 proteins reliably detected in this experiment, no additional protein was depleted $\left(P<0.05, \log _{2}\right.$ Ratio <1) (Fig. 4c, 4d). Notably, PROTAC(H-PGDS)-7 did not affect the protein levels of other PGs synthesis enzymes (PTGES2, PTGES3, AKR1B1) ${ }^{17-19}$ and CRBN target proteins (IZF1, IKZF3, ARID2, GSPT1, CSNK1A1) ${ }^{20-25}$ (Supplementary Dataset). We concluded that PROTAC(H-PGDS)-7-mediated protein degradation is highly specific to H-PGDS.

\section{Suppression of $\mathrm{PGD}_{2}$ and potent activity for $\mathrm{DMD}$ in vivo}

As discussed above, PROTAC(H-PGDS)-7 is a potent H-PGDS protein degrader that is dependent on the UPS. In addition to participating in H-PGDS degradation, PROTAC(HPGDS)-7 may also inhibit the enzymatic activity of H-PGDS; this is because it contains the 
TFC-007 moiety, which inhibits H-PGDS enzymatic activity. To investigate the importance of H-PGDS degradation on PGD 2 production, we used PROTAC(H-PGDS)-8 as a structurally related inactive form of the degrader but an active form of the inhibitor. We investigated the effect of PROTAC(H-PGDS)-7, PROTAC(H-PGDS)-8, and TFC-007 on the production of PGD 2 in KU812 cells. Treatment with PROTAC(H-PGDS)-7 for $6 \mathrm{~h}$ suppressed production of PGD 2 more effectively than TFC-007 and PROTAC(H-PGDS)-8 (Fig. 5). PROTAC(HPGDS)-8 and PROTAC(H-PGDS)-7 were considered to possess similar physical properties such as solubility and cell permeability because they have similar structures. PROTAC(HPGDS)-7 showed much stronger activity than PROTAC(H-PGDS)-8 to suppress production of $\mathrm{PGD}_{2}$, suggesting that PROTACs that act via a dual mechanism of enzyme inhibition and protein degradation might result in more potent effect than those that act through enzyme inhibition alone.

DMD is a severe $\mathrm{X}$-linked muscle disease caused by mutations in the dystrophin gene and ultimately results in cardiorespiratory dysfunction, which is likely the leading cause of death in patients ${ }^{26,27}$. It has been reported the overproduction of $\mathrm{PGD}_{2}$ by $\mathrm{H}-\mathrm{PGDS}$ is associated with muscle necrosis, inflammation, and muscle tissue damage in muscular dystrophy ${ }^{28}$. In addition, H-PGDS is expressed in myonecrotic areas in DMD patients ${ }^{29}$. Therefore, H-PGDS is a potential therapeutic target. Here, we investigated the effect of PROTAC(H-PGDS)-7 in vivo using a $m d x$ mouse model of DMD. Cardiac hypertrophy was induced in $m d x$ mice by thyroid hormone (T3)-treatment ${ }^{30}$, leading to upregulation of the cardiac mRNA levels of TNF $\alpha$ and IL-1 $\beta$ (a proinflammatory cytokine), TGF $\beta$ (a master regulator of fibrosis), and CD11b (a marker of macrophage and monocyte infiltration) (Fig. 6). The upregulation of these genes tended to be suppressed by treatment of PROTAC $(\mathrm{H}$ PGDS)-7 in $m d x$ mice with cardiac hypertrophy better than TFC-007 (Fig. 6). 


\section{Discussion}

In the design of PROTAC compounds, not only the appropriate combination of ligands for E3 ligase and the target protein, but also the appropriate linker length, greatly affects the degradation activity against the target protein. If the X-ray structure of the ternary complex is known, it is relatively easy to design an optimal PROTAC, but if not, it is always a matter of trial and error. On the other hand, we were able to prove that it is possible to design a potent PROTAC compound via in silico simulation of the ternary complex, as follows; we predicted that it would be possible to design a new PROTAC(H-PGDS) with a shorter linker due to the slack of linker in ternary complex with PROTAC(H-PGDS)-1, H-PGDS, and CRBN (Fig. 1). This hypothesis was supported by MD simulation of ternary complex with PROTAC $(\mathbf{H}$ PGDS)-3-6 with a shorten PEG linker and PROTAC(H-PGDS)-7 in which pomalidomide linking directly to TFC-007. The linker moiety in PROTAC compounds would have a Janusfaced effect on stability of the ternary complex. That is to say, the ternary complex can be stabilized if appropriate interactions such as hydrogen bonds can be formed among the amino acids of the protein and the linker moiety of PROTAC compounds, whereas the longer linker with high flexibility is easily exposed on the protein surface and is also enthalpically disadvantageous. In fact, the PEG moiety of a PROTAC(H-PGDS) with a longer linker was more easily exposed on the protein surface in our docking simulation (Fig. 1 and Extended Data Fig. 1). The difference of the orientation between H-PGDS and CRBN, i.e., PROTAC(HPGDS)-1 versus 7, changed the relationship in the stable ternary complex (Extended data Fig.3a, 3b), which possibly affects the approach of the entire E3 ligase complex to H-PGDS. One of the reasons for the potent activity of PROTAC(H-PGDS)-7 is that the linker moiety is not exposed to the solvent, which may have stabilized the energy of the ternary complex. In addition, it is possible that the interaction between H-PGDS and CRBN was altered by the linker length of PROTAC(H-PGDS)s, which may have affected the degrading activity of $\mathrm{H}$ PGDS (Extended data Fig.3a). As noted in the results, PROTAC(H-PGDS)-7 showed highest H-PGDS protein degradation activity $\left(\mathrm{DC}_{50}=17.3 \mathrm{pM}\right)$ among the designed PROTAC $(\mathbf{H}-$ 
PGDS)s. There are a limited number of PROTACs that can degrade target proteins at picomolar concentrations, such as them targeting $\mathrm{BET}^{31}$ and $\mathrm{FAK}^{32}$ protein. However, those PROTACs have been developed using various linkers through trial and error. In this study, we rationally designed such PROTACs with potent degradation activity based on in silico simulations and demonstrated their usefulness.

Currently, therapies for DMD are mainly two types: therapies for the restoration of dystrophin quantity, and therapies for the maintaining of muscle quality by suppression of the pathology caused by dystrophin deficiency ${ }^{33}$. Antisense oligonucleotides (ASOs) are a kind of the "quantity" therapies and recently four ASOs (eteplirsen, casimersen, golodirsen, vitolarsen) have been approved for the treatment of $\mathrm{DMD}^{34}$. Since the aim of the ASOs is to restore the reading frame of dystrophin transcripts, the applications are limited to DMD patients who have a confirmed mutation of dystrophin gene that is amenable to specific exon skipping. Also, the ASOs are approved an increase in dystrophin production in skeletal muscle but are not clear in cardiac muscle. On the other hand, glucocorticosteroids ${ }^{35}$ and $\mathrm{H}$ PGDS inhibitors ${ }^{36}$ are a kind of the "quality" therapies. Although the exact mechanism is not fully elucidated, they are expected to prevent the DMD pathology (such as inflammation), leading to delay disease progression in $\mathrm{DMD}$. For instance, $\mathrm{PGD}_{2}$-mediated inflammation is suggested to be involved in the development of muscle necrosis in DMD patients ${ }^{37}$ and $\mathrm{H}$ PGDS inhibitors have been demonstrated to tend to be suppressed the progression in DMD patients $^{15}$. To date, a variety of H-PGDS inhibitors has been developed, and a few has been tested in clinical trials ${ }^{36,38}$. However, these inhibitors have not performed satisfactorily in clinical studies likely because their pharmacokinetics and pharmacodynamics were not optimized. Therefore, it is expected that new clinical agents with a mechanism of action other than H-PGDS inhibition will be developed. The treatment of PROTAC(H-PGDS)-7 in $m d x$ mice with cardiac hypertrophy suppressed upregulation of the disease-related genes better than TFC-007(Fig. 6), indicating that PROTAC(H-PGDS)-7 would be a promising option in the "quality" therapy for cardiac hypertrophy in DMD patients. 
In addition, the linker can be shortened without losing activity, which reduces molecular weight of PROTAC(H-PGDS), and makes it more likely that a PROTAC(H-PGDS) would be a drug candidate for therapy of H-PGDS-related diseases. PROTACs are limited by their poor cell permeability and hence poor access to their intracellular targets, which may be related to their large molecular size, many rotatable bonds, and high hydrophobicity. ${ }^{39,40}$ From the comparison of druglikeness between PROTAC(H-PGDS)-1 and 7, the removal of the linker markedly reduces the molecular weight and rotatable bonds. The molecular weight of PROTAC(H-PGDS)-7 (MW: 743) is slightly lower than that of PROTAC(H-PGDS)-1 (MW: 1034), and reported PROTACs typically have molecular weights in the range from 900 to $1100^{39,40}$. While the number of rotatable bonds is preferred to be 10 or less following the rule of five ${ }^{41}$, typical PROTACs have bonds between 20 and $25^{42}$. PROTAC(H-PGDS)-7 has only 10 rotatable bonds, which is more appropriate for a drug than PROTAC(H-PGDS)-1, which has 28 rotatable bonds. The properties of PROTAC(H-PGDS)-7 are more in line with the rule of five chemical spaces than those of PROTAC(H-PGDS)-1, which suggests that the elimination of the linker moiety increases the likelihood that these complexes can be developed as drugs and is an efficient optimization method.

In conclusion, a potent degrader of the H-PGDS protein, PROTAC(H-PGDS)-7, was successfully developed by conjugating TFC-007 (H-PGDS ligand) to pomalidomide (E3 ligase, CRBN ligand). PROTAC(H-PGDS)-7 effectively induced the selective degradation of H-PGDS protein via the UPS and showed sustained suppression of $\mathrm{PGD}_{2}$ production. The degrader PROTAC(H-PGDS)-7 with its new mechanism of action is expected to be as effective or more effective than conventional inhibitors and may allow a reduction in the frequency of doses for the treatment of chronic inflammation. Additionally, the degrader showed more activity in vivo than that of TFC-007, which was expected to be clinically developed as an H-PGDS degrader. Further structure-based improvement of the H-PGDS degraders and evaluation of the safety, pharmacokinetics, and pharmacodynamics of our $\mathrm{H}$ PGDS degrader are in progress in our laboratory. 



\section{Methods}

\section{Design and synthesis of PROTAC(H-PGDS)}

The synthesis and physicochemical data on designated PROTAC(H-PGDS)s are provided in the Supplementary Information.

\section{Computational analysis}

The molecular dynamics (MD) simulations were performed using Molecular Operating Environment (MOE) 2020.09. All the simulations and analysis were calculated using Amber10: EHT force field. Glutathione was added to the conformation of docking simulation in the same pose as crystal structure (PDB: $5 \mathrm{YWX})^{43}$. Water molecules were added and the system was neutralized by addition of $\mathrm{NaCl}$ salt of $0.1 \mathrm{~mol} / \mathrm{L}$ concentration. A periodic boundary condition was utilized to carry out the simulation. In all cases, the cell size was larger than the protein by $10 \AA$. The temperature of the simulation was controlled by the NAMD. The initial energy minimization process of each simulation was performed by standard protocols in MOE 2020.09. Simulation speed was maintained 2 fs time step during all the simulations. The system was saved in every $0.5 \mathrm{ps}$ during simulations. First, $10 \mathrm{ps}$ time evolution was performed with a temperature $0 \mathrm{~K}$ (minimization step). Next, 100 ps time evolution was performed with a temperature $10 \mathrm{~K}$ to $300 \mathrm{~K}$ and tether $0.5 \AA$ to $100 \AA$ (equilibration step). Then, 100 ps time evolution was performed with a temperature $300 \mathrm{~K}$ (production step). After completion of MD simulation for all systems, the results were analyzed in MOE 2020.09. For each result, the most stable structure in production step was selected and calculated ligand interactions and potential energy.

\section{Reagents}

Tissue culture plastics were purchased from Greiner Bio-One (Frickenhausen, Germany). Cycloheximide was purchased from Sigma-Aldrich (St. Louis, MO, USA). MG132 was purchased from Peptide Institute (Osaka, Japan). MLN7243 was purchased from Active 
Biochem (Maplewood, NJ, USA). Pomalidomide was purchased from Cayman Chemical (Ann Arbor, MI, USA). A23187 was purchased from Merck KGaA (Darmstadt, Germany).

\section{Cell culture}

Human chronic myelogenous leukemia KU812 cells were cultured in Roswell Park Memorial Institute-1640 (RPMI-1640) medium (Sigma-Aldrich, St. Louis, MO, USA) supplemented with $10 \%$ fetal bovine serum (FBS) (Thermo Fisher Scientific, Waltham, MA, USA), and 100 $\mu \mathrm{g} / \mathrm{mL}$ kanamycin (Sigma-Aldrich). Human megakaryoblastic leukemia MEG-01s cells were cultured in Dulbecco's Modified Eagle's medium (Sigma-Aldrich) supplemented with 10\% FBS and $100 \mu \mathrm{g} / \mathrm{mL}$ kanamycin. KU812 cells were obtained from the Japanese Collection of Research Bioresources (Osaka, Japan) Cell Bank (JCRB0104) ${ }^{44}$.

\section{Western blot analysis}

Cells were lysed with SDS lysis buffer $(0.1 \mathrm{M}$ Tris- $\mathrm{HCl}$ at $\mathrm{pH} 8.0,10 \%$ glycerol, and $1 \%$ SDS). Protein concentration was measured by the BCA method (Thermo Fischer Scientific), and an equal amount of protein lysate was separated by SDS-PAGE, transferred to PVDF membranes (Millipore, County Cork, Ireland), and analyzed by western blot using the appropriate antibodies. The immunoreactive proteins were visualized using Clarity Western ECL substrate (Bio-Rad, Hercules, CA, USA), and their light emission was quantified with a LAS-3000 lumino-image analyzer (Fuji, Tokyo, Japan). The following antibodies were used: anti-H-PGDS rabbit polyclonal antibody $(\mathrm{pAb})^{45}$; anti-CyclinB1 rabbit pAb (\#12231) (Cell Signaling Technology, Danvers, MA, USA); anti- $\beta$-Actin mouse mAb (A2228) (Sigma-Aldrich).

\section{RNA isolation and quantitative PCR in KU812 cells}

Total RNA was prepared from cells using a RNeasy kit (Qiagen, Hilden, Germany). Firststrand cDNA was synthesized from $1 \mu \mathrm{g}$ of total RNA with an oligo-dT primer using the SuperScript First-Strand Synthesis System (Invitrogen, Carsbad, CA, USA). Quantitative 
real-time PCR was performed with an Applied Biosystems 7500 Fast Real-Time PCR system (Applied Biosystems, Foster City, CA, USA) using SYBR GreenER (Invitrogen) with genespecific primers. Human 36B4 mRNA was used as an invariant control. The following PCR primers were used $\left(5^{\prime}\right.$ to $\left.3^{\prime}\right)$ : 36B4, GGCCCGAGAAGACCTCCTT and CCAGTCTTGATCAGCTGCACA; H-PGDS, TGCCGTCGCTAACTGGATAA and GAGATGCCCCCGAGAAAAAC.

\section{Binding affinity to H-PGDS}

The fluorescence polarization-based (FP-based) binding assay was performed using Prostaglandin D Synthase (hematopoietic-type) FP-Based Inhibitor Screening Assay Kit Green (600007) (Cayman Chemical). In brief, the binding assays were performed in nonbinding black 384-well and used a recombinant human H-PGDS protein, glutathione and fluorescent probe in assay buffer to produce a final volume of $47.5 \mu \mathrm{l}$. Then, $2.5 \mu \mathrm{l}$ of test compounds made up as stocks in DMSO was added and the plate was incubated for $1 \mathrm{~h}$ at room temperature. Each was tested against H-PGDS in triplicate at final test compound concentrations $(2.50 \mu \mathrm{M}, 1.25 \mu \mathrm{M}, 625 \mathrm{nM}, 313 \mathrm{nM}, 156 \mathrm{nM}, 78.1 \mathrm{nM}, 39.0 \mathrm{nM}, 19.5 \mathrm{nM}$, $9.77 \mathrm{nM}, 4.88 \mathrm{nM}$ and $2.44 \mathrm{nM})$. Plates were then read with excitation wavelengths ( $470 \mathrm{~nm})$ and emission wavelengths $(530 \mathrm{~nm})$ on a EnVision Multilabel Plate Reader (PerkinElmer, Waltham, MA, USA). The measurements of fluorescent polarization of a molecule (mP) are taken in the fluorescent polarization mode. The percentage of inhibition of test compounds was calculated according to the following equation:

$$
\text { Percentage of inhibition }=\left(\frac{\mathrm{mP}_{100 \%}-\mathrm{mP}_{\text {sample }}}{\mathrm{mP}_{100 \%}}\right) \times 100
$$

where $\mathrm{mP}$ sample is the value of the wells containing test compounds, and $\mathrm{mP}_{100 \%}$ is the value of the maximum binding well. The concentration of test compounds that reduces the $\mathrm{mP}$ value by $50 \%\left(\mathrm{IC}_{50}\right)$ was estimated from a graph plotted the $\mathrm{mP}$ value versus the concentration of the compounds on semi-log axis. 


\section{TMTpro 16plex MS assay}

Cell lysates were prepared and digested by using EasyPep Mini MS Sample Prep kit (Thermo Fisher Scientific) according to manufacturer's protocol. $25 \mu \mathrm{g}$ of peptides from each sample were labelled with $0.25 \mathrm{mg}$ of TMTpro mass tag (TMT) labeling reagent (Thermo Fisher Scientific) according to manufacturer's protocol. After TMT labelling, the 16 sample channels were combined in an equal ratio, dried using a speed-vac, and resuspended in $0.1 \%$ TFA. Samples were fractionated into 8 fractions using High $\mathrm{pH}$ Reversed-Phase Peptide Fractionation Kit (Thermo Fisher Scientific) according to manufacturer's protocol. $1 \mu \mathrm{g}$ peptides from each fraction were analyzed by LC-MS/MS on EASY-nLC 1200-connected Orbitrap Fusion Lumos Tribrid mass spectrometer (Thermo Fisher Scientific) equipped with FAIMS-Pro ion mobility interface (Thermo Fisher Scientific). Peptides were separated on an analytical column $(\mathrm{C} 18,1.6 \mu \mathrm{m}$ particle size $\times 75 \mu \mathrm{m}$ diameter $\times 250 \mathrm{~mm}$, lon Opticks $)$ using $4 \mathrm{hr}$ gradients ( $0 \%$ to $28 \%$ acetonitrile over $240 \mathrm{~min}$ ) with a constant flow of $300 \mathrm{nl} / \mathrm{min}$. Peptides ionization was performed using Nanospray Flex Ion Source (Thermo Fisher Scientific). FAIMS-Pro was set to three phases (-40, -60 , and-80 CV) and a ' 1 sec cycle for a phase' data-dependent acquisition method was used where the most intense ions in every $1 \mathrm{sec}$ were selected for MS/MS fragmentation by HCD. MS raw files were analyzed using a Sequest HT search program in Proteome Discoverer 2.4 (Thermo Fisher Scientific). MS/MS spectra were searched against the SwissProt reviewed human reference proteome (UniProt). TMTpro-based protein quantification was performed using a Reporter lons Quantifier node in Proteome Discoverer 2.4.

\section{Measurement of $\mathrm{PGD}_{2}$ levels}

KU812 cells were treated with the indicated compounds for $6 \mathrm{~h}$, and stimulated with $5 \mu \mathrm{M}$ calcium ionophore A23187 (Sigma-Aldrich) for $10 \mathrm{~min}$. We measured PGD2, in the culture medium by LC/MS analysis. The deuterated internal standard $\mathrm{PGD}_{2}-\mathrm{d} 4$, (Cayman Chemical) 
was added to the collected medium. The method of sample purification was based on solid phase extraction with MonoSpin (GL Science, Tokyo, Japan). The $1000 \mu \mathrm{l}$ of sample was applied to the pre-activated cartridge, followed by washing the cartridge with $1 \mathrm{ml}$ of $2 \%(\mathrm{v} / \mathrm{v})$ acetic acid, followed by $700 \mu \mathrm{l}$ of hexane. The loaded samples were eluted with $500 \mu \mathrm{l}$ of ethyl acetate. The elute was evaporated, and the dried extracts were redissolved in $100 \mu \mathrm{l}$ of $1 \%(\mathrm{v} / \mathrm{v})$ methanol aqueous solution containing $0.1 \%(\mathrm{v} / \mathrm{v})$ formic acid. The resulting samples $(5 \mu \mathrm{l})$ were analyzed using liquid chromatography (LC-20AD UFLC system, Shimadzu, Kyoto, Japan) coupled to a triple quadrupole mass spectrometer (LCMS-8040, Shimadzu). A chromatographic separation was performed on an STR-ODS- II column $(2.0 \times$ $150 \mathrm{~mm}$, Shinwa Chemical, Kyoto, Japan) at $40^{\circ} \mathrm{C}$. The mobile phases consisted of a linear gradient of water $(A)$ and acetonitrile $(B)$ containing both $0.1 \%(v / v)$ formic acid: $A: B=70: 30$ $(\mathrm{v} / \mathrm{v})$ at $0 \mathrm{~min} ; 20: 80(\mathrm{v} / \mathrm{v})$ at $10 \mathrm{~min}$. The flow rate was $300 \mu \mathrm{l} / \mathrm{min}$. The effluent from the column was measured by mass spectrometry using electrospray ionization (ESI). ESI parameters were as follows: DL temperature $250^{\circ} \mathrm{C}$; heat-block temperature $400{ }^{\circ} \mathrm{C}$; drying gas flow $15 \mathrm{~L} / \mathrm{min}$ and $\mathrm{ESI}$ voltage $3.5 \mathrm{kV}$. The mass spectrometer was operated in a multiple reaction monitoring negative ionization mode. For analysis, the monitored transition for $\mathrm{PGD}_{2}$ was $\mathrm{m} / \mathrm{z} 351 \rightarrow 271$ and for $\mathrm{PGD}_{2}$-d4 it was $\mathrm{m} / \mathrm{z} 355 \rightarrow 275$. Elution time for $\mathrm{PGD}_{2}$ was identified by elution time: 5.5 minutes.

\section{In vivo analysis}

Nine-weeks-old to twelve-weeks-old male $m d x$ mice, dystrophin-deficient, generous gift from Dr. Shin'ichi Takeda (National Center of Neurology and Psychiatry, Japan) were used in this study. The mice were housed and maintained at an ambient temperature of $22 \pm 0.5^{\circ} \mathrm{C}$ with a relative humidity of $50 \pm 2 \%$ and with an automatically controlled 12-hr light/12-hr dark cycle (lights on at 8:00 a.m., illumination intensity of $\approx 100$ lux). The mice were allowed free access to laboratory chow and water until the start of experiment. Animal experiments were carried out humanely in accordance with the Regulations for Animal Experiments of the Daiichi 
University of Pharmacy and Fundamental Guidelines for Proper Conduct of Animal Experiment and Related Activities in Academic Research Institutions under the jurisdiction of the Ministry of Education, Culture, Sports, Science, and Technology of Japan and with approval from the Institutional Animal Experiment Committee of Daiichi University of Pharmacy.

Cardiac hypertrophy was induced in $m d x$ mice by thyroid hormone [3,3',5-triiodo-Lthyronine (T3), Sigma-Aldrich $]^{30}$. T3 was dissolved in $0.1 \mathrm{~N} \mathrm{NaOH}$, and brought to $\mathrm{pH} 8-9$ with HCl. PROTAC(H-PGDS)-7 or TFC-007 was suspended in physiological saline containing 1\% DMSO. T3 (2 mg/kg/day) in the presence or absence of PROTAC(H-PGDS)-7 (45 $\mathrm{mg} / \mathrm{kg} /$ day) or TFC-007 (30 mg/kg/day) was injected subcutaneously into $m d x$ mice for 14 days. Total RNA was extracted from mouse heart by the guanidinium thiocyanate-phenolchloroform method using ISOGEN (Nippon Gene, Tokyo, Japan). Quantitative real-time PCR was performed with a Light-Cycler amplification and detection system (Roche Diagnostics, Indianapolis, IN), using FastStart Essential DNA Green Master (Roche) with gene-specific primers. Murine GAPDH mRNA was used as an invariant control. The following PCR primers were used (5' to $\left.3^{\prime}\right)$ : GAPDH, TGAACGGGAAGCTCACTGG and TCCACCACCCTGTTGCT; TNF $\alpha$ AGTGACAAGCCTGTAGCCCACG and TTTCTCCTGGTATGAGATAGC; IL-1 $\beta$, TTGACGGACCCCAAAAGATG and AGAAGGTGCTCATGTCCTCA; TGF- $\beta 1$, TGCGCTTGCAGAGATTAAAA and AGCCCGAAGCGGACTACTAT; CD11b, CAGGGACAACCACACCTCTTG and GCAGCGTCATACCAGCACAC.

\section{Acknowledgements}

This study was supported in part by grants from the Japan Agency for Medical Research and Development (21ak0101073j0905 to Y.D.); Japan Society for the Promotion of Science and the Ministry of Education, Culture, Sports, Science and Technology (JSPS/MEXT KAKENHI Grants Number JP20K22711 to H.Y., JP18K07311 to N.S., JP20K06568 to A.E., 
JP17K08385 and 21K05320 to Y.D., JP18H05498 to Y.S., and JP18H05502 to M.N. and Y.D.); Takeda Science Foundation (to N.S. and Y.D.); the Naito Foundation (to H.Y. and Y.D.); the Sumitomo Foundation (to Y.D.); the Novartis Foundation (Japan) for the Promotion of Science (to Y.D.), Japan Foundation of Applied Enzymology (to Y.D.), Kobayashi Foundation for Cancer Research (to Y.D.), the High-Quality Protein Crystal Growth Experiment on JEM promoted by Japan Aerospace Exploration Agency (JAXA) (to K.A.), and Foundation for Promotion of Cancer Research in Japan (to Y.D.). We would like to express their deepest appreciation to Dr. Kentaro Kamiya (MOLSIS Inc.) for his help in the PROTAC modeling. We thank Ms. Y. Hosokawa undergraduate student of Daiichi University of Pharmacy for excellent technical assistance. We thank Renee Mosi, PhD, from Edanz (https://jp.edanz.com/ac) for editing a draft of this manuscript.

\section{Authors Contributions}

H.Y., N.S., K.A., M.N., and Y.D. designed the research, and H.Y., N.S., and Y.D wrote the paper. H.Y., N.S., A.E., T.I., Y.Y., Y.M., K.F., Y.S., and K.A. performed the experiments and analyzed results. All authors discussed the results and commented on the manuscript.

\section{Competing Interests statement}

The Authors declare that they have no conflict of interest. 


\section{References}

1. Lai, A.C. \& Crews, C.M. Induced protein degradation: an emerging drug discovery paradigm. Nat. Rev. Drug Discov. 16, 101-114 (2017).

2. Burslem, G.M. \& Crews, C.M. Proteolysis-Targeting Chimeras as Therapeutics and Tools for Biological Discovery. Cell 181, 102-114 (2020).

3. Wang, Y., Jiang, X., Feng, F., Liu, W. \& Sun, H. Degradation of proteins by PROTACs and other strategies. Acta. Pharm. Sin. B. 10, 207-238 (2020).

4. Gadd, M.S. et al. Structural basis of PROTAC cooperative recognition for selective protein degradation. Nat. Chem. Biol. 13, 514-521 (2017).

5. Nowak, R.P. et al. Plasticity in binding confers selectivity in ligand-induced protein degradation. Nat. Chem. Biol. 14, 706-714 (2018).

6. Testa, A., Hughes, S.J., Lucas, X., Wright, J.E. \& Ciulli, A. Structure-Based Design of a Macrocyclic PROTAC. Angew. Chem. Int. Ed. Engl. 59, 1727-1734 (2020).

7. Drummond, M.L. \& Williams, C.I. In Silico Modeling of PROTAC-Mediated Ternary Complexes: Validation and Application. J. Chem. Inf. Model. 59, 1634-1644 (2019).

8. Drummond, M.L., Henry, A., Li, H. \& Williams, C.I. Improved Accuracy for Modeling PROTAC-Mediated Ternary Complex Formation and Targeted Protein Degradation via New In Silico Methodologies. J. Chem. Inf. Model. 60, 5234-5254 (2020).

9. Zaidman, D., Prilusky, J. \& London, N. PRosettaC: Rosetta Based Modeling of PROTAC Mediated Ternary Complexes. J. Chem. Inf. Model. 60, 4894-4903 (2020).

10. Bai, N. et al. Rationalizing PROTAC-Mediated Ternary Complex Formation Using Rosetta. J. Chem. Inf. Model. 61, 1368-1382 (2021).

11. Lewis, R.A. et al. Prostaglandin D2 generation after activation of rat and human mast cells with anti-lgE. J. Immunol. 129, 1627-1631 (1982).

12. Matsuoka, T. et al. Prostaglandin D2 as a mediator of allergic asthma. Science 287, 2013-2017 (2000). 
13. Urade, Y. \& Hayaishi, O. Biochemical, structural, genetic, physiological, and pathophysiological features of lipocalin-type prostaglandin D synthase. Biochim. Biophys. Acta. 1482, 259-271 (2000).

14. Takeshita, E. et al. A phase I study of TAS-205 in patients with Duchenne muscular dystrophy. Ann. Clin. Transl. Neurol. 5, 1338-1349 (2018).

15. Komaki, H. et al. Early phase 2 trial of TAS-205 in patients with Duchenne muscular dystrophy. Ann. Clin. Transl. Neurol. 7, 181-190 (2020).

16. Yokoo, H. et al. Development of a Hematopoietic Prostaglandin D SynthaseDegradation Inducer. ACS Med. Chem. Lett. 12, 236-241 (2021).

17. Hara, S. et al. Prostaglandin E synthases: Understanding their pathophysiological roles through mouse genetic models. Biochimie 92, 651-659 (2010).

18. Kabututu, Z. et al. Prostaglandin F2alpha synthase activities of aldo-keto reductase 1B1, 1B3 and 1B7. J. Biochem. 145, 161-168 (2009).

19. Watanabe, K. Recent reports about enzymes related to the synthesis of prostaglandin (PG) F(2) (PGF(2alpha) and 9alpha, 11beta-PGF(2)). J. Biochem. 150, 593-596 (2011).

20. Kronke, J. et al. Lenalidomide causes selective degradation of IKZF1 and IKZF3 in multiple myeloma cells. Science 343, 301-305 (2014).

21. Lu, G. et al. The myeloma drug lenalidomide promotes the cereblon-dependent destruction of Ikaros proteins. Science 343, 305-309 (2014).

22. Gandhi, A.K. et al. Immunomodulatory agents lenalidomide and pomalidomide costimulate $\mathrm{T}$ cells by inducing degradation of $\mathrm{T}$ cell repressors Ikaros and Aiolos via modulation of the E3 ubiquitin ligase complex CRL4(CRBN.). Br. J. Haematol. 164, 811-21 (2014).

23. Yamamoto, J. et al. ARID2 is a pomalidomide-dependent CRL4(CRBN) substrate in multiple myeloma cells. Nat. Chem. Biol. 16, 1208-1217 (2020).

24. Matyskiela, M.E. et al. A novel cereblon modulator recruits GSPT1 to the CRL4(CRBN) ubiquitin ligase. Nature 535, 252-257 (2016). 
25. Kronke, J. et al. Lenalidomide induces ubiquitination and degradation of CK1alpha in del(5q) MDS. Nature 523, 183-188 (2015).

26. Connuck, D.M. et al. Characteristics and outcomes of cardiomyopathy in children with Duchenne or Becker muscular dystrophy: a comparative study from the Pediatric Cardiomyopathy Registry. Am. Heart J. 155, 998-1005 (2008).

27. Benditt, J.O. \& Boitano, L. Respiratory support of individuals with Duchenne muscular dystrophy: toward a standard of care. Phys. Med. Rehabil. Clin. N. Am. 16, 1125-1139 (2005).

28. Mohri, I. et al. Inhibition of prostaglandin D synthase suppresses muscular necrosis. Am. J. Pathol. 174, 1735-1744 (2009).

29. Okinaga, T. et al. Induction of hematopoietic prostaglandin D synthase in hyalinated necrotic muscle fibers: its implication in grouped necrosis. Acta Neuropathol. 104, 377384 (2002).

30. Anderson, J.E., Liu, L. \& Kardami, E. The effects of hyperthyroidism on muscular dystrophy in the mdx mouse: greater dystrophy in cardiac and soleus muscle. Muscle Nerve 17, 64-73 (1994).

31. Raina, K. et al. PROTAC-induced BET protein degradation as a therapy for castrationresistant prostate cancer. Proc. Natl. Acad. Sci. U S A 113, 7124-7129 (2016).

32. Gao, H. et al. Design, Synthesis, and Evaluation of Highly Potent FAK-Targeting PROTACs. ACS Med. Chem. Lett. 11, 1855-1862 (2020).

33. Duan, D., Goemans, N., Takeda, S., Mercuri, E. \& Aartsma-Rus, A. Duchenne muscular dystrophy. Nat. Rev. Dis. Primers 7, 13 (2021).

34. Fortunato, F., Rossi, R., Falzarano, M.S. \& Ferlini, A. Innovative Therapeutic Approaches for Duchenne Muscular Dystrophy. J. Clin. Med. 10(2021).

35. Angelini, C. \& Peterle, E. Old and new therapeutic developments in steroid treatment in Duchenne muscular dystrophy. Acta Myol. 31, 9-15 (2012). 
36. Rittchen, S. \& Heinemann, A. Therapeutic Potential of Hematopoietic Prostaglandin D2 Synthase in Allergic Inflammation. Cells 8(2019).

37. Nakagawa, T. et al. A prostaglandin D2 metabolite is elevated in the urine of Duchenne muscular dystrophy patients and increases further from 8 years old. Clin. Chim. Acta. 423, 10-14 (2013).

38. Thurairatnam, S. Hematopoietic prostaglandin D synthase inhibitors. Prog. Med. Chem. 51, 97-133 (2012).

39. Doak, B.C., Over, B., Giordanetto, F. \& Kihlberg, J. Oral druggable space beyond the rule of 5: insights from drugs and clinical candidates. Chem. Biol. 21, 1115-1142 (2014).

40. DeGoey, D.A., Chen, H.J., Cox, P.B. \& Wendt, M.D. Beyond the Rule of 5: Lessons Learned from AbbVie's Drugs and Compound Collection. J. Med. Chem. 61, 2636-2651 (2018).

41. Veber, D.F. et al. Molecular properties that influence the oral bioavailability of drug candidates. J. Med. Chem. 45, 2615-2623 (2002).

42. Maple, H.J., Clayden, N., Baron, A., Stacey, C. \& Felix, R. Developing degraders: principles and perspectives on design and chemical space. Med. Chem. Comm. 10, 1755-1764 (2019).

43. Takaya, D. et al. Characterization of crystal water molecules in a high-affinity inhibitor and hematopoietic prostaglandin D synthase complex by interaction energy studies. Bioorg. Med. Chem. 26, 4726-4734 (2018).

44. Kishi, K. A new leukemia cell line with Philadelphia chromosome characterized as basophil precursors. Leuk. Res. 9, 381-390 (1985).

45. Mohri, I., Eguchi, N., Suzuki, K., Urade, Y. \& Taniike, M. Hematopoietic prostaglandin D synthase is expressed in microglia in the developing postnatal mouse brain. Glia $\mathbf{4 2}$, 263-274 (2003). 


\section{Figure Legends}

Fig. 1. (a) Chemical structures of PROTACs against H-PGDS and TFC-007. (b) Ternary complex of PROTAC(H-PGDS)-1, H-PGDS (green), and CRBN (orange). (c) Ternary complex of PROTAC(H-PGDS)-7, H-PGDS (green), and CRBN (orange).

Fig. 2. Comparison of the H-PGDS protein reduction activity of PROTAC(H-PGDS)-1, 3-7. KU812 cells were incubated with the indicated concentration of compounds for $6 \mathrm{~h}(\mathbf{a}, \mathbf{b})$, and $24 \mathrm{~h}(\mathbf{c})$. The H-PGDS/ $\beta$-Actin ratios were normalized by the vehicle control as 100 . Data in the bar graph are the means $\pm \operatorname{SEM}(n=3) .{ }^{*} P<0.05$ compared with DMSO-treated control in a Dunnett's test. Uncropped gels for $\mathbf{a}$ and $\mathbf{b}$ are included as Source Data.

Fig. 3. Mechanism of PROTAC(H-PGDS)-7-induced degradation of the H-PGDS protein. (a) KU812 cells were incubated with 100 pM PROTAC(H-PGDS)-7 or the ligand mixture (TFC007 and pomalidomide, $100 \mathrm{pM}$ each) for $6 \mathrm{~h}$. (b) Competition assay using an excess amount of pomalidomide with PROTAC(H-PGDS)-7 in KU812 cells. Cells were incubated with 100 pM PROTAC(H-PGDS)-7 and/or $1 \mu \mathrm{M}$ pomalidomide for 6 h. (c) Fluorescence polarization assays of the binding affinity between H-PGDS and TFC-007, PROTAC(H-PGDS)-7, and PROTAC(H-PGDS)-8. (d) KU812 cells were incubated with the indicated concentration of PROTAC(H-PGDS)-8 for 6 h. (e) Effect of MG132 and MLN7243 on the protein knockdown activity of PROTAC(H-PGDS)-7 in KU812 cells. Cells were incubated with 100 pM PROTAC(H-PGDS)-7 in the presence or absence of $10 \mu \mathrm{M}$ MG132 or $10 \mu \mathrm{M}$ MLN7243 for $6 \mathrm{~h}$. The H-PGDS/ $\beta$-actin ratios were normalized by the vehicle control as 100 . The data in the bar graphs are means \pm SEM $(n=3) .{ }^{*} P<0.05$ compared with the DMSO-treated control in a Dunnett's test. Uncropped gels for $\mathbf{a}, \mathbf{b}, \mathbf{d}$, and $\mathbf{e}$ are included as Source Data.

Fig. 4. TMTpro-based proteome analysis of KU812 cells treated with PROTAC(H-PGDS)-7.

(a) Experimental flows are shown (see also Method section in Supporting Information). 
KU812 cells treated with PROTAC(H-PGDS)-7 were subjected to LC-MS/MS analysis. (b) H-PDGS protein abundances were scaled by setting the mean value in 16 samples to 100 . The individual values, mean, and SEM from 2 or 4 biological replicates are shown. (c, d) The mean $\log _{2}$ abundance ratio (100 pM PROTAC(H-PGDS)/DMSO) (c) or the mean $\log _{2}$ abundance ratio (10 nM PROTAC(HPGDS)/DMSO) (d), and - $\log _{10}(P$-value) are indicated in the $x$ and $y$ axes, respectively $(n=4)$.

Fig. 5. Effect of PROTAC(H-PGDS)-7 on the production of $\mathrm{PGD}_{2}$ in $\mathrm{KU} 812$ cells. Cells were incubated with the indicated concentration of compounds for $6 \mathrm{~h}$. Then, the cells were incubated with $5 \mu \mathrm{M}$ A23187 in the presence of each compound for $10 \mathrm{~min}$. $\mathrm{PGD}_{2}$ levels in the medium were measured. The data in the bar graph are means \pm SEM $(n=3) .{ }^{*} P<0.05$ compared with the DMSO-treated control using a Dunnett's test.

Fig. 6. Effect of PROTAC(H-PGDS)-7 on cardiac hypertrophy in $m d x$ mice. $m d x$ mice were injected subcutaneously with T3 (2 mg/kg/day) in the presence or absence of PROTAC(HPGDS)-7 (45 mg/kg/day) or TFC-007 (30 mg/kg/day) for 2 weeks. The mRNA levels for TNF $\alpha$, IL-1 $\beta$ TGF $\beta$, and CD11b were measured by quantitative RT-PCR. The data in the bar graphs are the means \pm SEM $(n=3-5) .{ }^{*} P<0.05,{ }^{* *} P<0.01$ compared with the T3-treated control in a Dunnett's test.

Extended Data Fig. 1. Top pose of ternary complexes of each PROTAC(H-PGDS)-1 and 37. CRBN or H-PGDS was shown in orange and green respectively. Those complexes contained GSH at ligand binding cavity of H-PGDS.

Extended Data Fig. 2. Crucial amino acids 2D interaction between H-PGDS (PDB: 5YWX) and CRBN (PDB: 4Cl3) and the proposed compounds where (a, d) is for PROTAC(HPGDS)-4, (b, e) for PROTAC(H-PGDS)-6 and (c, f) for PROTAC(H-PGDS)-7 before (a-c) or 
after (d-f) MD simulation (100 ps), respectively. The proximity contour (dashed lines) and solvent exposed areas (solid purple spheres) of the ligand atoms are indicated, as are the polar (pink), hydrophobic (green), and solvent-exposed (light blue shadow) binding pocket amino acids. Acidic and basic residues are highlighted with red and blue halos, respectively.

Extended Data Fig. 3. (a) Overlay of two ternary complexes. A ternary complex of CRBN (dark orange), H-PGDS (dark green) and PROTAC(H-PGDS)-1 and other ternary complex of CRBN (light orange), H-PGDS (light green) and PROTAC(H-PGDS)-7 were aligned at each CRBN. (b) Ligands of ternary complexes such as PROTAC(H-PGDS)-1 (blue), PROTAC(H-PGDS)-3 (cyan), PROTAC(H-PGDS)-4 (green), PROTAC(H-PGDS)-5 (yellow), PROTAC(H-PGDS)-6 (orange) and PROTAC(H-PGDS)-7 (magenta). (c, d) The distance between the imide moiety of pomalidomide and the H380 of CRBN (c) or the pyrimidine moiety of TFC-007 and the W104 of H-PGDS (d) calculated from the simulations of the ternary complexes containing PROTAC(H-PGDS)-7 (cyan) and PROTAC(H-PGDS)-9 (magenta).

Extended Data Fig. 4. PROTAC(H-PGDS)-7 reduced H-PGDS protein levels in MEG-01s cells. Cells were treated with the indicated concentration of PROTAC(H-PGDS)-7 for $6 \mathrm{~h}$. The H-PGDS/ $\beta$-Actin ratios were normalized by vehicle control. Data in the bar graph are means \pm SEM $(n=3) .{ }^{*} P<0.05$ compared with vehicle-treatment control in a Dunnett's test. Uncropped gels are included as Source Data.

Extended Data Fig. 5. PROTAC(H-PGDS)-VH did not affect H-PGDS protein levels in KU812 cells. Cells were treated with the indicated concentration of PROTAC(H-PGDS)-VH for $6 \mathrm{~h}$. The H-PGDS/ $\beta$-Actin ratios were normalized by vehicle control. Data in the bar graph are means $\pm \operatorname{SEM}(n=3)$. Uncropped gels are included as Source Data. 
Extended Data Fig. 6. Chemical structures of the H-PGDS inhibitors F092 and TFC-007 and X-ray crystal structure of H-PGDS with F092 and glutathione (PDB: 5YWX).

Extended Data Fig. 7. (a) Fluorescence polarization assays of the binding affinity between H-PGDS and TFC-007, PROTAC(H-PGDS)-9, and 11 ( $n=3)$. TFC-007, PROTAC(H-PGDS)9 and compound 11 showed similar affinity toward H-PGDS with $\mathrm{IC}_{50}$ values of $0.68,1.1$ and $2.8 \mu \mathrm{M}$, respectively. (b) Fluorescence polarization assays of the binding affinity between $\mathbf{H}$ PGDS and TFC-007, PROTAC(H-PGDS)-10 ( $n=3)$. TFC-007 and PROTAC(H-PGDS)-10 showed similar affinity toward $\mathrm{H}-\mathrm{PGDS}$ with $\mathrm{IC}_{50}$ values of 0.14 and $0.17 \mu \mathrm{M}$, respectively.

Extended Data Fig. 8. PROTAC(H-PGDS)-9 did not affect H-PGDS protein levels in KU812 cells. Cells were treated with the indicated concentration of PROTAC(H-PGDS)-9 for $6 \mathrm{~h}$. The H-PGDS/ $\beta$-Actin ratios were normalized by vehicle control. Data in the bar graph are means $\pm \operatorname{SEM}(n=3)$. Uncropped gels are included as Source Data.

Extended Data Fig. 9. PROTAC(H-PGDS)-10 reduced H-PGDS protein levels in KU812 cells. Cells were treated with the indicated concentration of PROTAC(H-PGDS)-10 for $6 \mathrm{~h}$. The H-PGDS/ $\beta$-Actin ratios were normalized by vehicle control. Data in the bar graph are means \pm SEM $(n=3) .{ }^{*} P<0.05$ compared with vehicle-treatment control in a Dunnett's test. Uncropped gels are included as Source Data.

Extended Data Fig. 10. Turnover of H-PGDS protein in KU812 cells. (a) Cells were treated with $10 \mathrm{mg} / \mathrm{mL}$ cycloheximide $(\mathrm{CHX})$ in the presence or absence of 100 pM PROTAC(HPGDS)-7 for the indicated periods. H-PGDS/ $\beta$-Actin ratios were normalized by vehicle control. (b) Levels of H-PGDS mRNA in KU812 cells. Cells were treated with the indicated concentration of PROTAC(H-PGDS)-7 for 6 h. H-PGDS mRNA levels are relative to vehicle treatment, which was arbitrarily set to 1 . Data in the graphs are means $\pm \operatorname{SEM}(n=3)$. ${ }^{*} P<$ 
0.05 compared with vehicle-treatment control in a Dunnett's test. Uncropped gels for a are included as Source Data. 